\title{
Shifting sands: the role of radiotherapy for patients with gastric and gastroesophageal adenocarcinoma
}

\author{
Ethan B. Ludmir, Prajnan Das \\ The University of Texas MD Anderson Cancer Center, Houston, TX, USA \\ Contributions: (I) Conception and design: All authors; (II) Administrative support: None; (III) Provision of study materials or patients: None; (IV) \\ Collection and assembly of data: None; (IV) Collection and assembly of data: All authors; (V) Data analysis and interpretation: All authors; (VI) \\ Manuscript writing: All authors; (VII) Final approval of manuscript: All authors. \\ Correspondence to: Prajnan Das, MD, MS, MPH. 1400 Pressler St., Unit 1422, Houston, TX 77030, USA. Email: prajdas@mdanderson.org.
}

\begin{abstract}
Gastric adenocarcinoma, a leading cause of cancer-related mortality worldwide, is treated primarily with surgical resection in the non-metastatic setting. However, the optimal role and sequencing of adjunctive therapies, including radiotherapy (RT) as well as systemic therapy, remains unclear. A complex milieu of trials spanning several decades has evaluated different treatment strategies for gastric cancer, including the role of RT. In this review, we summarize the trial-level evidence for the diverse gastric cancer treatment paradigms. Despite initial success, postoperative RT has not shown a clear benefit in modern prospective studies in the setting of more aggressive surgical nodal dissection. On the other hand, the role of preoperative RT in optimizing oncologic outcomes for gastric cancer patients remains relatively underexplored; ongoing trials assessing preoperative RT aim to illuminate the optimal treatment strategy for nonmetastatic gastric cancer patients.
\end{abstract}

Keywords: Radiotherapy; gastric cancer; gastroesophageal cancer; preoperative; postoperative

Received: 18 February 2020; Accepted: 11 March 2020; Published: 25 July 2021.

doi: $10.21037 / \operatorname{tgh} .2020 .03 .01$

View this article at: http://dx.doi.org/10.21037/tgh.2020.03.01

\section{Introduction}

Gastric cancer is a leading cause of cancer-associated mortality worldwide (1). While the incidence and mortality associated with gastric cancers steadily declined over the past half-century, the epidemiological and oncologic underpinnings of these trends remain complex $(2,3)$. Gastric cancer incidence is highest in East Area, with relatively high rates also observed in Eastern Europe as well as South America (4). The differential incidence of the disease globally is in many ways a function of the risk factors for gastric adenocarcinoma (GAC), the most common (>90\%) histologic subtype of gastric cancer and the subject of this review $(2,5)$. As discussed in more detail elsewhere, the risk factors for gastric adenocarcinoma include Helicobacter pylori infection, ingestion of smoked foods, obesity, gastroesophageal reflux disease (GERD), and tobacco use, among others (2). These risk factors are associated with lesions occurring in different parts of the stomach: gastric cardia and gastroesophageal junction (GEJ) tumors are associated with obesity, smoking, and GERD, whereas gastric body and antral lesions are associated with $H$. pylori infection and chronic atrophic gastritis (2). Additionally, the genomic landscape of GAC varies based on site of lesion within the stomach; the molecular subtypes of GAC correlate with anatomic location of the primary tumor (6). Owing to the high incidence of GAC in East Asia, GAC screening programs have been implemented in countries such as Japan (2). Questions have been raised regarding whether such screening efforts have led to improved survival through early detection of gastric cancers in Japan, or if the observed differences in survival between screening and non-screening countries is due to overdiagnosis (7). These considerations notwithstanding, gastric cancer remains the third-leading cause of cancer-related death worldwide, and the optimal treatment strategy for this challenging disease 
remains opaque (8).

At the core of the treatment strategy for non-metastatic GAC is surgical resection. For select superficial, small lesions, endoscopic resection alone may be curative (5,9-11). For more invasive tumors (AJCC $8^{\text {th }}$ edition T1b and greater lesions), definitive resection conventionally consists of either a total or subtotal gastrectomy (subtotal considered for more distal tumors, in general) $(11,12)$. The extent of nodal dissection associated with gastrectomy is an area of active discussion, and is highly relevant in considering the role of radiotherapy (RT) in the treatment of gastric cancer. For the purposes of this discussion, the key types of dissection are D1 and D2 lymphadenectomy; D1 dissections involve removal of perigastric nodes, whereas D2 dissections include removal of nodes at the celiac axis and its branches (including nodes along the left gastric, common hepatic, celiac trunk, splenic hilum, and splenic artery) (13). Multiple randomized controlled trials (RCTs) across disparate patient populations have sought to determine any oncologic benefit to increased extent of nodal dissection (14-20). Individual RCTs comparing D1 and D2 dissection have not demonstrated an overall survival (OS) advantage to more extended dissection (14-17,20). However, the largest two of these trials, both conducted in Western populations (the Dutch and MRC trials), demonstrated that D2 dissection was associated with a more-than-double rate of postoperative mortality $(14,16)$. Despite this, long-term results highlight comparable OS rates between the D1 and D2 arms in these trials, suggesting that D2 dissection may improve disease-related survival if post-operative mortality could be minimized $(15,17)$. Indeed, long-term results of the Dutch trial show decreased rates of GAC-related death (37\% versus $48 \%$ ) in the D2 arm (15). Meta-analyses of D1 versus D2 GAC trials has similarly demonstrated that diseasespecific survival (DSS), but not OS or disease-free survival (DFS), is improved with D2 dissection (21). Given concerns that perioperative mortality may be obscuring a potential oncologic benefit of D2 dissection, the experience of the treating surgeon has been evaluated; studies have shown that center volume, surgeon experience, and dissection of at least 15 nodes (pathologically-confirmed) are associated with improved outcomes (22-24). Notably, more extended dissections beyond D2 (such as dissection of para-aortic nodes) has not been shown to demonstrate any oncologic benefit, even among experienced surgeons with low perioperative mortality $(<1 \%)(18,19)$. Putting the pieces together, D2 dissection appears to be the optimal surgical management for regional nodes among GAC patients, but should be performed in the context of an experienced center/surgeon with low perioperative mortality. As we shall see, surgical management of GAC patients, and in particular extent of lymphadenectomy, factor heavily into considerations regarding the role of RT.

Owing to the poor outcomes for GAC patients, even those treated with adequate surgical resection, consideration has long been given to adjuvant and neoadjuvant therapies. Decades-old patterns of relapse data demonstrated that GAC patients treated with surgery alone had primarily distant metastatic recurrence, with a large component of local and regional relapse as well (25). Therefore, both systemic adjuvant therapy (chemotherapy) and locoregional adjuvant therapy (RT) have been evaluated in series of RCTs. These have been studied in the preoperative, postoperative, and, for systemic therapy, perioperative settings. In this review, we discuss the role of RT in the treatment of GAC in the context of two distinct treatment paradigms-postoperative and preoperative RT. Advances in RT techniques to improve the therapeutic ratio are reviewed, and the future of RT in the broader landscape of gastric cancer treatment is considered.

\section{Postoperative}

The seminal trial demonstrating a benefit of postoperative RT is the Intergroup 0116 (INT-0116) RCT, which accrued patients in the 1990s (26,27). In this cooperative group trial (based in the United States), patients with nonmetastatic gastric or GEJ adenocarcinoma were treated with definitive surgical resection followed by randomization to either observation or adjuvant chemoradiation (CRT) (26). The postoperative CRT regimen consisted of 45Gy RT in 25 daily fractions, delivered using 2 opposed [anteroposterior/ posteroanterior (AP/PA)] beams designed to encompass the tumor bed as well as regional nodes including the perigastric, celiac, splenic, hepatoduodenal, and local paraaortic basins (26). Chemotherapy was delivered using a regimen of 5-fluouracil (5-FU) and leucovorin, delivered as 1 cycle before RT, 2 cycles concurrent with RT, and 2 cycles post-RT $(26,28)$. Eligibility criteria included stage IBIV gastric or GEJ adenocarcinoma (approximately $20 \%$ of enrolled patients had GEJ tumors), and R0 (margin-negative) resection (26); the primary endpoint (PEP) of the trial was OS. The trial results demonstrated significant improvements in OS, relapse-free survival, and local relapse with adjuvant CRT (Table 1) (26,27). With long-term follow-up, the trial highlighted an approximate $10 \%$ benefit in OS with 
Table 1 Major trials of postoperative RT for gastric cancer

\begin{tabular}{|c|c|c|c|c|c|c|c|c|c|}
\hline Trial & Phase & $\begin{array}{l}\text { Country/ } \\
\text { countries }\end{array}$ & Randomization & $\mathrm{N}$ & $\begin{array}{l}\text { RT } \\
\text { technique }\end{array}$ & $\begin{array}{l}\mathrm{D} 2 \\
\text { dissection } \\
\text { rate }\end{array}$ & $\mathrm{pN}+$ rate & DFS/RFS/EFS & OS \\
\hline ARTIST-2 & III & South Korea & $\begin{array}{l}\text { Postoperative SOX + RT } \\
\text { (45 Gy) vs. SOX vs. S-1 } \\
\text { (3-arm) }\end{array}$ & 538 & $\begin{array}{l}\text { Not } \\
\text { reported }\end{array}$ & $100 \%$ & $100 \%$ & $\begin{array}{l}73 \% \text { vs. } 78 \% \text { vs. } 65 \% \\
(\mathrm{P}=0.67 \text { for } \mathrm{SOX}+\mathrm{RT} \\
\text { vs. SOX), 3-year }\end{array}$ & Not reported \\
\hline CRITICS & III & $\begin{array}{l}\text { Netherlands, } \\
\text { Sweden, } \\
\text { Denmark }\end{array}$ & $\begin{array}{l}\text { Postoperative CRT } \\
\text { (45 Gy + capecitabine + } \\
\text { cisplatin) vs. EOC/ECC }\end{array}$ & 788 & $\begin{array}{l}\text { 3D or IMRT } \\
\text { allowed }\end{array}$ & $6.8 \%$ & $\begin{array}{l}51.1 \% \\
(\mathrm{ypN}+)\end{array}$ & $\begin{array}{l}38 \% \text { vs. } 39 \% \\
(P=0.92), 5 \text {-year }\end{array}$ & $\begin{array}{l}40 \% \text { vs. } 42 \% \\
(P=0.90), 5 \text {-year }\end{array}$ \\
\hline
\end{tabular}

$\mathrm{RT}$, radiotherapy; $\mathrm{N}$, number; $\mathrm{pN}+$, pathologically node-positive; LR, local relapse; DFS, disease-free survival; RFS, relapse-free survival; EFS, event-free survival; OS, overall survival; INT, Intergroup; CRT, chemoradiation; 5-FU, 5-fluouracil; LCV, leucovorin; AP, anteroposterior; PA, posteroanterior; XPRT, capecitabine/cisplatin plus radiotherapy; XP capecitabine/cisplatin; EOC, epirubicin/oxaliplatin/capecitabine; ECC, epirubicin/cisplatin/capecitabine; IMRT, intensity-modulated radiotherapy; ypN+, pathologically-node-positive after neoadjuvant therapy.

adjuvant CRT (27). INT-0116 accrued a large proportion of more locoregionally-advanced GAC patients; about twothirds of enrolled patients had pT3/T4 tumors, and $85 \%$ of patients had pathologically node-positive disease. The trial further stipulated that those patients with hepatic metastases were considered to have regional rather than distant metastatic disease (26). It is also important to note that the above benefits in disease-related outcomes (DROs) with the addition of adjuvant CRT were observed despite the older RT technique (AP/PA two-dimensional RT) and associated increased toxicity. Only 64\% of CRT-arm patients completed the prescribed adjuvant therapy, and $32 \%$ of patients experienced a grade 4 toxicity with CRT (26). Finally, a critical component in interpreting the results of this trial is the extent of nodal dissection, which was not mandated in the trial protocol nor included in the postoperative randomization stratification scheme (although D2 dissection was "recommended" in the protocol) (26). Only $10 \%$ of patients in INT-0116 underwent D2 dissection, with $54 \%$ and $36 \%$ undergoing D0 and D1 dissections, respectively (26). In the context of such high rates of suboptimal nodal dissection, interpretation of INT-0116 is complex. On long-term follow-up of the trial results, differential benefit of adjuvant CRT was not observed based on extent of nodal dissection; however, this was an unplanned subgroup analysis with few $(\mathrm{n}=54)$ patients in the
D2 subgroup across arms (27). This same subgroup analysis, however, did reveal trends toward differential benefit of postoperative therapy based on both sex and tumor histology, with both women and diffuse-histology patients experiencing less benefit from CRT than men or intestinalhistology patients (27). Collectively, INT-0116 set the stage for the GAC approach primarily used in the United States, favoring adjuvant CRT.

Given the limited nodal dissection for INT-0116 patients, the role of postoperative RT in the context of a more aggressive nodal dissection remained in question. Three further large-scale RCTs have attempted to address this question: ARTIST, ARTIST-2, and CRITICS (29-34). The ARTIST and ARTIST-2 trials, both conducted in South Korea, evaluated the role of adjuvant RT for patients status post D2 dissection and R0 resection, with planned adjuvant chemotherapy in both the control and experimental arms $(29,31,33,35)$. In the ARTIST trial, patients were randomized postoperative to either 6 cycles of adjuvant capecitabine/cisplatin (XP; control arm), or XP with RT (XPRT; experimental arm) (29,35). For the XPRT arm, patients received 2 cycles of XP, followed by 45 Gy RT (in 25 daily fractions) concurrent with capecitabine (twicedaily $825 \mathrm{mg} / \mathrm{m}^{2}$ ), and then 2 further cycles of XP (29). RT was delivered using AP/PA fields, with comparable target volumes as per INT-0116 (29). In the analysis of the PEP 
(DFS), there was no improvement with the addition of RT to XP, although a trend toward improved DFS was noted with XPRT on both the initial and long-term reports of the trial results (Table 1) $(29,34)$. Notably, a large proportion of patients had earlier-stage disease, with approximately $60 \%$ of patients having stage IB-II disease; therefore, the observed event rate was lower than anticipated, and the authors contend that this may obscure a potential signal seen for the experimental arm (29). On long-term followup, subgroup analysis demonstrated that pathologically node-positive patients and patients with intestinal-type histology patients may disproportionately benefit from XPRT over XP (34). In both INT-0116 and ARTIST, those patients with diffuse-type GAC were less likely to benefit from adjuvant RT on post-hoc subgroup analysis; notably, only $39 \%$ of patients in INT-0116 had diffuse histology, compared with $60 \%$ of patients in ARTIST $(26,27,34)$. Given the predilection for diffuse-type GAC for distant metastatic spread, the lack of benefit with adjunctive locoregional therapy for this subgroup of patients is logical (36). It further helps to contextualize the benefit of RT seen in INT-0116, and absence of benefit seen in ARTIST. Lastly, the treatment compliance was improved from INT-0116 to ARTIST, with $75 \%$ of the XP arm and $82 \%$ of the XPRT arm completing the prescribed adjuvant therapy (29,35). Together, ARTIST did not convincingly demonstrate a benefit of adjuvant RT to adjuvant XP-based chemotherapy in an unselected GAC population status post $\mathrm{D} 2$ dissection and $\mathrm{R} 0$ resection.

The findings of ARTIST spurred ARTIST-2, a three-arm RCT evaluating the role of adjuvant therapy approaches among $\mathrm{R} 0$-resected D2-dissected pathologically-node-positive GAC patients. The poorer oncologic outcomes of pathologically-node-positive patients, and the ARTIST-identified potential benefit of $\mathrm{RT}$ in this subgroup, resulted in the ARTIST-2 trial design and eligibility criteria. However, whereas ARTIST focused on the role of RT in the setting of adjuvant XP-based chemotherapy, ARTIST-2 compared S-1 and SOX (S-1 plus oxaliplatin) chemotherapy $(31,33)$. This shift in adjuvant systemic therapy backbone is based on the Japanese ACTSGC study, in which stage II-III GAC patients status post D2 dissection and R0 resection were randomized to adjuvant S-1 versus observation (37). Adjuvant S-1 was found to improve both relapse-free survival (RFS) and OS (37). Consequently, ARTIST-2 randomized patients to adjuvant S-1 for 12 months, adjuvant SOX for 6 months, or adjuvant RT to 45Gy plus SOX (6 months; SOX+RT), with DFS as the PEP. Reported in abstract form in 2019, the results demonstrate that $\mathrm{SOX}$ or $\mathrm{SOX}+\mathrm{RT}$ were more effective in prolonging DFS when compared to adjuvant S-1 alone (Table 1) (31). However, there appeared to be no difference between the SOX and SOX+RT arms, with 3 -year DFS being $78 \%$ and $73 \%$, respectively (31). While full publication of results is still pending at the time of this writing, these data, broadly-speaking, temper enthusiasm for adjuvant RT in the context of even pathologically-nodepositive patients status post $\mathrm{D} 2$ dissection. Absent published results, a number of critical details regarding enrollment and results remain unknown, such as proportion of patients with diffuse versus intestinal histology tumors. The ARTIST-2 trialists, in light of subgroup analyses of both INT-0116 and ARTIST, included histologic subtype as a stratification factor for ARTIST-2; results stratified by tumor histology are anticipated pending publication of full results $(31,33)$. That said, the presented subgroup analyses by the trialists did not reveal any convincing patient subgroup that may differentially benefit from adjuvant SOX+RT rather than SOX (31). Pooling the results and key discussion points for both ARTIST and ARTIST-2, there appears to be a limited role for adjuvant RT for patients having undergone $\mathrm{R} 0$ resection with $\mathrm{D} 2$ dissection, particularly as advances in systemic therapies continue to improve oncologic outcomes.

While ARTIST and ARTIST-2 evaluated postoperative RT for Korean patients having undergone D2 dissection, the CRITICS trial modified the relevant oncologic questions in its efforts to ascertain the role of adjuvant RT for GAC (32). Rather than recommending a D2 dissection, the CRITICS trialists mandated patients undergo a D1+ dissection, representing resection of at least 15 pathologically-confirmed nodes with removal of all N1 (D1) nodes as well as N2 nodal stations 7-9 and 11 (left gastric, common hepatic, celiac axis, and splenic arterial) $(23,32,38)$. The $\mathrm{D} 1+$ dissection paradigm, which omits splenopancreatectomy as compared with the D2 dissection (which includes both splenectomy and distal pancreatectomy), aims to preserve the oncologic benefits of D2 dissection while minimizing surgical morbidity and mortality $(15,21,32,39)$. Increased postoperative mortality was associated with splenic and pancreatic resections as part of the standard D2 dissection, and a Japanese RCT demonstrated non-inferior oncologic outcomes for resected proximal GAC patients randomized to splenic preservation versus splenectomy $(15,21,39)$. Therefore, the CRITICS trialists, following a model suggested by the Dutch D1 versus D2 dissection RCT group, mandated 
D1+ dissection for enrolled GAC patients. Furthermore, the CRITICS trialists attempted to evaluate the role of adjuvant RT against the European MAGIC regimen (40). The MAGIC trial, performed by the MRC in the United Kingdom, demonstrated that perioperative chemotherapy with epirubicin/cisplatin/5-FU (3 cycles preoperatively and 3 cycles postoperatively) improved OS compared with resection alone (40). Based on MAGIC, patients enrolled on the CRITICS trial received 3 cycles of preoperative epirubicin/cisplatin/capecitabine (ECC) or epirubicin/ oxaliplatin/capecitabine (EOC) followed by resection with at least $\mathrm{D} 1+$ dissection; postoperative treatment of patients was randomized between a control arm of EOC/ECC for 3 further cycles (as per the MAGIC trial) versus an experimental arm of 45 Gy RT (in 25 daily fractions) with concurrent cisplatin and capecitabine (32). The study PEP for CRTICS was OS. In the reported results of the trial, there was no OS or event-free survival (EFS) advantage with postoperative CRT rather than postoperative chemotherapy (Table 1) (32). Pre-specific post-hoc subgroup analyses by histology, tumor location, gender, and age did not reveal any notable trends with regard to differential treatment benefit with postoperative CRT (32). As patients were randomized at the start of all therapy (prior to preoperative chemotherapy), post-hoc analyses were not reported based on surgical pathologic features such as pathological-node-positive (ypN+) status following preoperative chemotherapy (41). As approximately half of patients in CRITICS were ypN+ on surgical pathology, the role of adjuvant RT for these higher-risk patients is of interest, particularly in the context of the negative ARTIST-2 trial $(31,32,38,42)$.

Importantly, the CRITICS trial highlights an important aspect of adjuvant therapy-treatment compliance and toxicity. While patients across both arms received over $90 \%$ of the recommended chemotherapeutic dose intensities of preoperative ECC/EOC, only $46 \%$ of patients in the postoperative chemotherapy arm and $50 \%$ of patients in the postoperative CRT arm completed the allocated treatment as initially planned (32). Postoperative RT fields, for both gastric and GEJ lesions, are larger and associated with higher toxicity rates than preoperative fields (43). Organs in the RT field with preoperative therapy are typically resected with subsequent surgery, whereas organs in-field in the setting of postoperative RT include the anastomotic site and a greater volume of bowel. The benefit of preoperative RT with regard to treatment compliance as well as toxicity has been shown for other gastrointestinal malignancies, most notably rectal cancer (44). In the context of esophageal cancer as well, attempts at postoperative RT have not been successful, with high rates of RT-related toxicity (including grade 5 toxicity) $(45,46)$. In contrast, for rectal as well as esophageal and GEJ lesions, preoperative therapy has become standard of care, strongly supported by level 1 evidence $(44,47)$. Therefore, the successor CRITICS-II trial is evaluating the role of preoperative RT, described in more detail below (48).

\section{Preoperative}

Improved treatment compliance and reduced toxicity favor the use of preoperative therapy. In addition, preoperative therapy, particularly preoperative RT, has the potential to downstage lesions and improve the odds of complete (R0) resection; this has been shown in the MAGIC trial with perioperative chemotherapy as described above (40). Yet, the use of perioperative chemotherapy in MAGIC resulted in no patients with pathologic complete response (pCR) (40). In contrast, use of preoperative RT has the potential to yield more substantial pathological downstaging, including pCR, as demonstrated in esophageal and GEJ lesions with the CROSS trial (47). The CROSS trial, which provides among the strongest pieces of level 1 evidence supporting preoperative CRT for esophageal/GEJ tumors, randomized patients to either definitive surgical resection or preoperative CRT with concurrent carboplatin/paclitaxel and 41.4 Gy RT (delivered in 23 fractions) followed by surgery (47). Patients on the CROSS trial largely had adenocarcinoma histology tumors (75\%), and approximately a quarter of CROSS patients had GEJ lesions (Table 2) (47). Remarkably, patients treated with preoperative CRT had markedly higher rates of R0 resection compared with the surgery-alone cohort (92\% vs. 69\%) (47). Furthermore, the pCR rate for all preoperative CRT patients was $29 \%$, and for adenocarcinoma patients was $23 \%$, in contrast to the absence of $\mathrm{pCR}$ observed in the perioperative chemotherapy patients in MAGIC $(40,47)$. With a PEP of OS, the CROSS trial demonstrated a significant benefit of preoperative CRT, with a median OS of 49.4 vs. 24.0 months (47). Notably, although the study was not powered to assess the treatment effect by histology, this benefit appeared to be less pronounced among patient with adenocarcinomas, with an adjusted hazard ratio for preoperative CRT benefit of 0.74 (95\% confidence interval 0.54-1.02, $\mathrm{P}=0.07$ ) (47). No subgroup analysis by tumor location (i.e., for GEJ lesions) has been provided to date. Subset analyses notwithstanding, 
Table 2 Major trials of postoperative RT for gastric and GEJ cancer

\begin{tabular}{|c|c|c|c|c|c|c|c|c|c|c|}
\hline Trial & Phase & Country & Tumor site & Randomization & $\mathrm{N}$ & RT Technique & $\begin{array}{l}\text { D2 } \\
\text { Dissection } \\
\text { Rate }\end{array}$ & $\begin{array}{l}\text { Ro } \\
\text { Rate }\end{array}$ & $\begin{array}{l}\mathrm{pCR} \\
\text { Rate }\end{array}$ & OS \\
\hline $\begin{array}{l}\text { RTOG } \\
9904\end{array}$ & II & USA & $\begin{array}{l}\text { Gastric and } \\
\text { GEJ }\end{array}$ & $\begin{array}{l}\text { Single-arm; all treated } \\
\text { with induction ChT } \\
(5-F U / \text { leucovorin/ } \\
\text { cisplatin) } \rightarrow \text { CRT (45 Gy + } \\
\text { 5-FU/taxol) } \rightarrow \text { Sx }\end{array}$ & $\begin{array}{r}49 \\
+ \\
+\end{array}$ & 3D-CRT & $50 \%$ & $77 \%$ & $26 \%$ & $72 \%, 1$-year \\
\hline
\end{tabular}

RT, radiotherapy; GEJ, gastroesophageal junction; N, number; R0, margin-negative resection; pCR, pathological complete response; OS, overall survival; CRT, chemoradiation; carbo/taxol, carboplatin/paclitaxel; Sx, surgery; 3D-CRT, 3-dimensional conformal radiotherapy; ChT, chemotherapy; cis/etop, cisplatin/etoposide; PLF, cisplatin/5-FU/leucovorin; taxol, paclitaxel.

CROSS results demonstrate a $7 \%$ grade 3 toxicity rate in the preoperative CRT arm, $>90 \%$ compliance with the preoperative CRT regimen, and a $94 \%$ rate of subsequent surgery in the CRT arm. These empirically demonstrate the advantages of the preoperative approach with improved compliance, decreased toxicity, and assessment of tumor response (47). In light of the CROSS study, a strong case is therefore made for approaching GEJ lesions with a preoperative CRT paradigm.

It is worth noting, though, that despite the striking findings of the CROSS study in support of preoperative CRT for esophageal and GEJ tumors, CROSS compared preoperative CRT to no adjunctive therapy (surgery alone). The POET trial, on the other hand, specifically examined those patients with GEJ adenocarcinoma (locallyadvanced, uT3-4 Nx M0) and compared treatment with preoperative chemotherapy (cisplatin/5-FU/leucovorin) alone (2.5 'courses') versus preoperative chemotherapy (same regimen for 2 courses) followed by CRT (to 30 Gy in 15 daily fractions with concurrent cisplatin / etoposide), both followed by surgery $(49,50)$. While the POET trial was closed due to poor accrual, only accruing 126 of 354 intended patients, the trial demonstrated higher rates of pCR with CRT rather than chemotherapy alone (16\% vs. $2 \%$ ) (Table 2). Despite the low statistical power of the trial, OS appeared to be improved with the preoperative CRT approach (3-year OS $47 \%$ vs. $26 \%, \mathrm{P}=0.06)(49,50)$. Local control similarly appeared to be improved with the addition of preoperative CRT (49). While the interpretation of
POET is limited by poor accrual, on face it lends support to utilization of preoperative CRT for GEJ lesions, consistent with the CROSS study. However, we would be remiss if we did not note that two contemporaneous trials for esophageal/GEJ adenocarcinoma comparing preoperative chemotherapy vs. CRT did not demonstrate or suggest long-term disease-control benefits $(51,52)$. While the nuances of these largely-esophageal trials is beyond the scope of this review, it is worth noting that the recommended chemotherapy regimens continue to evolve, and consideration has been made for whether differences in surgical technique may account for the observed differences in outcomes between these trials (53). As both surgery and RT are locally-directed therapies, the interplay between these in the context of esophageal and gastric tumors is critical in interpreting trial results (53). All this said, the CROSS trial, as well as the POET study, lend support to utilization of preoperative CRT for GEJ adenocarcinomas. This approach has become the predominant treatment paradigm for GEJ lesions in the United States, as well as our home institution (54).

While this discussion regarding preoperative RT has thus far centered on the relatively abundant literature for esophageal and GEJ cancers, less has been reported for gastric adenocarcinomas. So much so, that no published phase III data have been reported evaluating preoperative RT for GAC. The seminal experience for this treatment paradigm among GAC patients is the RTOG 9904 trial (55). This single-arm multi-institutional phase II cooperative 
group trial in the United States evaluated the impact of preoperative therapy on pathologic surrogate endpoints (namely pCR) (55). Patients were treated with induction chemotherapy with 2 cycles of 5-FU/leucovorin/cisplatin followed by concurrent CRT (45 Gy in 25 daily fractions, with concurrent $5-\mathrm{FU} /$ paclitaxel) and then resection (D2 encouraged but not required) (Table 2) (55). Half of RTOG 9904 patients underwent D2 dissection, 77\% underwent R0 resection, and $26 \%$ experienced a pCR. Twelve-month OS was improved among those patients with pCR (82\% vs. $69 \% ; 72 \%$ for the full cohort) (55). By exceeding the a priori pCR threshold of $20 \%$, RTOG 9904 presented a total preoperative paradigm of induction chemotherapy followed by preoperative CRT as a viable strategy for GAC patients. This strategy has been tested in other phase I/II studies (56-61). Some have shown similar pCR rates as with RTOG 9904 as well as CROSS $(47,55-57,60,61)$, and others have shown high rates of conversion of locally-advanced unresectable primary site disease into margin-negative resectable disease (56). Our institutional treatment paradigm has centered on the use of induction chemotherapy followed by preoperative CRT. Retrospective efforts from our center have shown that preoperative-CRT-based approaches do not compromise surgical morbidity or mortality $(62,63)$, and are associated with high rates of subsequent completion surgery $(64,65)$. The importance of post-treatment nodal pathological status (ypN status) in particular has been highlighted by our group using the preoperative CRT approach $(66,67)$.

Yet, while feasibility and efficacy have been shown for the preoperative CRT paradigm in these smaller experiences (up to single-arm phase II studies), there are a few critical caveats to note. Most importantly, absent randomized prospective data to validate the preoperative CRT approach, the generalizability of the above data remains challenging; the efficacy of a particular treatment approach, particularly one where the expertise of the treating surgeon and radiation oncologist are integral, makes extrapolation of the above data limited. Furthermore, use of surrogate pathological endpoints such as pCR may not reflect treatment efficacy per se, but rather may simply 'unmask' different strata of GAC patients based on prognosis or treatment-responsiveness. This may be beneficial unto itself as it may allow for postoperative treatment intensification, such as with systemic therapies (including targeted therapies, immunotherapy, and similar). Patients with pCR or ypN0 disease may be able to defer treatment intensification, while those with incomplete pathological response or ypN+ disease may warrant further therapy adjuvantly. Irrespective, absent phase III data, the utility of preoperative CRT for large-scale implementation across centers remains unclear; the use of preoperative CRT nationwide in the United States, for instance, has been declining over time (54).

Meanwhile, the use of perioperative chemotherapy has been widely accepted for GAC patients. This strategy, described above for the MAGIC trial, has now been further augmented with the publication of the FLOT trial results $(40,68)$. In the FLOT trial (a phase II/III randomized controlled study in Germany), 716 patients were randomized to perioperative epirubicin-containing chemotherapy similar to the MAGIC study (control arm) versus perioperative FLOT chemotherapy (docetaxel/ oxaliplatin/leucovorin/5-FU; experimental arm) (68). The trial demonstrated superior OS (the PEP) with the FLOT regimen, with 3 -year OS $57 \%$ in the FLOT arm versus $48 \%$ in the control arm (68). FLOT was further associated with a higher rate of $\mathrm{R} 0$ resection $(85 \%$ vs. $78 \%$ ) and higher rate of pathological downstaging than the epirubicincontaining control regimen (ypN0 49\% vs. $41 \%$; pCR not reported in the primary publication of the trial) (68). Despite this survival advantage, there was no significant difference in toxicity between the two regimens, confirming the new standard of care for perioperative chemotherapy with FLOT (68).

As perioperative chemotherapy continues to advance and is increasingly utilized, the role of RT is called into question. To adequately assess the role of RT for GAC, particularly in the preoperative setting, phase III data are required. Several ongoing trials aim to answer this core question. The TOPGEAR trial (NCT01924819) is randomizing GAC patients to perioperative epirubicinbased chemotherapy (a la MAGIC) versus preoperative CRT (in which 2 cycles of epirubicin-based chemotherapy are given followed by 45 Gy CRT with concurrent 5 -FU or capecitabine); patients are then to undergo D1+ dissection and postoperatively patients in both arms are treated with 3 cycles of adjuvant epirubicin-based chemotherapy $(69,70)$. The study's PEP is OS, with robust quality of life secondary endpoints (70). Interim results from the first 120 patients accrued to TOPGEAR have shown high rates of treatment compliance with the preoperative regimens in both arms, without increased toxicity or postoperative morbidity associated with preoperative CRT (69). While results are pending, TOPGEAR is limited by the results of the FLOT trial above, which redefined FLOT 
as the standard of care chemotherapeutic regimen for perioperative systemic therapy for nonmetastatic GAC patients. That said, TOPGEAR has been amended in light of the FLOT trial results, incorporating the new regimen into the trial design; statistical power considerations and post hoc analyses based on these different systemic therapy regimens may complicate the interpretation of TOPGEAR results. In addition to TOPGEAR, the CRITICS-II trial (NCT02931890) is randomizing GAC patients to one of three different preoperative therapy approaches (48). Patients will be randomized to preoperative FLOT-like chemotherapy ("DOC", consisting of docetaxel/oxaliplatin/ capecitabine) alone for 4 cycles, DOC for 2 cycles followed by CRT (45 Gy in 25 daily fractions with concurrent paclitaxel/carboplatin, similar to the CROSS regimen), or CRT (as above, similar to CROSS) alone $(47,48,68)$. All patients are then to undergo D2 dissection, without adjuvant therapy; the PEP of the trial is 1-year EFS (48). The purpose of the three-arm CRITICS-II phase II study is to identify the "most promising" regimen to subsequently compare in phase III investigation (48). The CRITICSII trial incorporates elements from much of the above discussion, assessing the role of RT in the context of a D2 dissection, as well as an improved systemic therapy regimen with FLOT-like chemotherapy. Along the same lines, for GEJ (and esophageal) tumors, the ESOPEC trial (NCT02509286) is assessing the role of perioperative FLOT versus CROSS-like preoperative CRT (71). Results of these trials, which have the potential to illuminate the optimal treatment approach for gastric and GEJ adenocarcinoma patients, are eagerly awaited.

\section{RT techniques}

The bulk of discussion thus far has revolved around triallevel data guiding overarching treatment strategies for GAC patients. These trials have spanned many decades, and during this time RT-based techniques and technologies have advanced profoundly, complicating the application of historical RT-related results to the modern era. The evolution of RT techniques, from two-dimensional AP/ PA fields (2D) to three-dimensional conformal RT (3D-CRT) to intensity-modulated RT (IMRT) and beyond is of significant consequence with regard to the toxicities associated with RT delivery (72-76). Irrespective of the preoperative $v s$. postoperative context, gastric-cancerdirected RT carries high risk of radiation-related toxicity. Historical results from INT-0116, in which patients were treated postoperatively to 45 Gy in 25 daily fractions with 2D RT using AP/PA fields with concurrent 5-FU/ leucovorin, noted that only $64 \%$ of patients completed the postoperative CRT regimen as prescribed, and $17 \%$ of patients discontinued CRT due to toxicity (26). A majority of INT-0116 patients experienced grade 3+ hematologic toxicity with CRT, and one third of patients experienced grade 3+ gastrointestinal toxicity (26). As noted above, postoperative RT is generally associated with higher risk of RT-related toxicity; postoperative fields encompass a larger area than preoperative fields, and the irradiated organs are not surgically resected in the weeks following RT. With the evolution from 2D RT (AP/PA) to 3D-CRT in which dose homogeneity could be improved [described in more detail elsewhere (73-75)], and the transition to preoperative therapy, RTOG 9904 demonstrated still-high rates of severe toxicity, with $21 \%$ of patients experiencing grade 4+ adverse events with preoperative CRT (55). The RT volumes themselves, discussed above, conventionally include the stomach (or tumor bed plus anastomosis in the postoperative setting) as well as the perigastric, celiac, splenic, and hepatoduodenal basins; some variations on these volumes may be considered depending on the primary tumor location. For GEJ/cardia tumors, for instance, omission of subpyloric, suprapyloric, and pancreaticoduodenal nodes can be considered. For antral/ pyloric lesions, on the other hand, coverage of the splenic artery/splenic hilar basins may not be necessary.

With the advent of computed tomography (CT) and CTbased RT treatment planning, delineation of target volumes allowed for specification of precisely which structures warranted inclusion in the clinical target volume. From this, IMRT developed using inverse-planning algorithms; multiple beams (often 8 or more) would be utilized to provide a more conformal treatment plan in which prespecified dose constraints and target volume coverage could be optimized $(72,76)$. Dosimetric studies comparing 3D-CRT and IMRT for gastric cancer demonstrated potential advantages with IMRT through dose reduction to the liver, kidneys, heart, and spinal cord $(77,78)$. Other studies were not as enthusiastic, suggesting that modest dosimetric improvements to the kidneys were unlikely to provide clinical benefit in the setting of gastric cancer RT fields (79). Owing to the low-dose radiation dose bath associated with IMRT (and related techniques such as volumetric-modulated arc therapy, or VMAT), IMRT may not spare surrounding luminal gastrointestinal structures from radiation dose, and therefore may not improve the 
largely GI-based RT toxicity profile (79).

Comparative clinical studies, while limited to retrospective experiences, suggests some benefit with use of IMRT in the preoperative or postoperative settings. In the preoperative setting, a recent report from our institution compared 82 patients treated with 3D-CRT versus 120 patients treated with IMRT; IMRT was associated with lower rates of grade 3-4 acute toxicity (49\% vs. $70 \%$ ), and lower rates of treatment-related events (56\% vs. $85 \%$ ) (72). Notably, lymphopenia was also improved among IMRT-treated patients (72). In the postoperative setting, reports have shown decreased treatment breaks with IMRT, as well as decreased risk of late nephrotoxicity with IMRT $(80,81)$. With this in mind, it is worth noting that the ongoing CRITICS-II trial mandates use of IMRT/VMAT in the preoperative treatment of GAC patients, ensuring that the CRITICS-II trial reflects modern RT techniques (48).

Despite the observed clinical benefits of IMRT, overall RT-related toxicity even in the preoperative setting remains substantial. Future efforts may focus on improving the therapeutic ratio with the use of RT for GAC through use of increasingly-advanced technologies as well as more refined target volumes. Technologies such as proton beam therapy or magnetic-resonance-guided RT (i.e., through use of a magnetic-resonance-imaging-equipped linear accelerator) may decrease unnecessary radiation exposure of uninvolved normal structures. On the other hand, the target volumes defined for GAC RT as above may warrant reconsideration. While peer-reviewed publication of final results of the trial are pending, ARTIST-2 seems to have shown that $\mathrm{D} 2$-dissected $\mathrm{pN}+$ patients do not seem to benefit from adjuvant RT (31). It is conceivable that preoperative RT for GAC may shift toward local fields directed at the primary tumor and involved nodal regions only; certainly it is worth noting that RT fields directed at the primary tumor and involved nodes only (without treatment of elective nodal region) may change interpretation of post-treatment pathological nodal status $(66,67,82)$. Yet, while primary-tumor-directed RT may drastically reduce the RT field size and associated toxicity, the question of how RT affects patterns of failure calls this strategy into question $(82,83)$. RT-related improvements in regional nodal control may contribute toward an overall oncologic benefit associated with RT $(66,67,82-84)$. These questions and many others remain unanswered with regard to the optimal RT target volumes and treatment technique, and merit further exploration.

\section{Conclusions}

The role of RT in the treatment of GAC and GEJ adenocarcinoma has been a moving target for decades, with a complex milieu of trial results highlighting a diverse array of potential treatment strategies for these challenging malignancies. Postoperative RT for GAC, among the best studied RT-inclusive treatment paradigms, has not shown a clear benefit in modern trials, pending final results of ARTIST-2. While select subsets of GAC patients may benefit from postoperative RT, attention has shifted to preoperative therapy. Promising results from smaller prospective efforts has led to a number of ongoing later-phase trials, including CRITICS-II and TOPGEAR. Whether modern RT techniques in the preoperative setting provide oncological benefit to patients remains to be seen, particularly in light of successive improvements with the perioperative chemotherapy approach seen in MAGIC and now FLOT. Standing on shifting sands of evidence and standards of care, parsing through the results of these anticipated trials will hopefully provide clarity on the optimal approach for treatment of non-metastatic gastric cancer patients.

\section{Acknowledgments}

Funding: None.

\section{Footnote}

Provenance and Peer Review: This article was commissioned by the Guest Editor (Jaffer A. Ajani) for the series "Gastrointestinal Cancer" published in Translational Gastroenterology and Hepatology. The article has undergone external peer review.

Conflicts of Interest: Both authors have completed the ICMJE uniform disclosure form (available at http://dx.doi. org/10.21037/tgh.2020.03.01). The series "Gastrointestinal Cancer" was commissioned by the editorial office without any funding or sponsorship. PD reports personal fees from Adlai Nortye, personal fees from MD Anderson Cancer Center Madrid, Spain, outside the submitted work. The authors have no other conflicts of interest to declare.

Ethical Statement: The authors are accountable for all aspects of the work in ensuring that questions related to the accuracy or integrity of any part of the work are appropriately investigated and resolved. 
Open Access Statement: This is an Open Access article distributed in accordance with the Creative Commons Attribution-NonCommercial-NoDerivs 4.0 International License (CC BY-NC-ND 4.0), which permits the noncommercial replication and distribution of the article with the strict proviso that no changes or edits are made and the original work is properly cited (including links to both the formal publication through the relevant DOI and the license). See: https://creativecommons.org/licenses/by-nc-nd/4.0/.

\section{References}

1. Torre LA, Siegel RL, Ward EM, et al. Global Cancer Incidence and Mortality Rates and Trends--An Update. Cancer Epidemiol Biomarkers Prev 2016;25:16-27.

2. Karimi P, Islami F, Anandasabapathy S, et al. Gastric cancer: descriptive epidemiology, risk factors, screening, and prevention. Cancer Epidemiol Biomarkers Prev 2014;23:700-13.

3. Bray F, Ren JS, Masuyer E, et al. Global estimates of cancer prevalence for 27 sites in the adult population in 2008. Int J Cancer 2013;132:1133-45.

4. Forman D, Burley VJ. Gastric cancer: global pattern of the disease and an overview of environmental risk factors. Best Pract Res Clin Gastroenterol 2006;20:633-49.

5. Ajani JA, Lee J, Sano T, et al. Gastric adenocarcinoma. Nat Rev Dis Primers 2017;3:17036.

6. Cancer Genome Atlas Research Network. Comprehensive molecular characterization of gastric adenocarcinoma. Nature 2014;513:202-9.

7. Bollschweiler E, Boettcher K, Hoelscher AH, et al. Is the prognosis for Japanese and German patients with gastric cancer really different? Cancer 1993;71:2918-25.

8. Bray F, Ferlay J, Soerjomataram I, et al. Global cancer statistics 2018: GLOBOCAN estimates of incidence and mortality worldwide for 36 cancers in 185 countries. CA Cancer J Clin 2018;68:394-424.

9. Gotoda T, Yanagisawa A, Sasako M, et al. Incidence of lymph node metastasis from early gastric cancer: estimation with a large number of cases at two large centers. Gastric Cancer 2000;3:219-25.

10. Gotoda T. Endoscopic resection of early gastric cancer. Gastric Cancer 2007;10:1-11.

11. Ajani JA, D'Amico TA, Almhanna K, et al. Gastric Cancer, Version 3.2016, NCCN Clinical Practice Guidelines in Oncology. J Natl Compr Canc Netw 2016;14:1286-312.

12. Amin MB, American Joint Committee on Cancer., American Cancer Society. AJCC cancer staging manual.
Chicago IL, American Joint Committee on Cancer, Springer, 2017.

13. Japanese Gastric Cancer Association. Japanese gastric cancer treatment guidelines 2014 (ver. 4). Gastric Cancer 2017;20:1-19.

14. Bonenkamp JJ, Hermans J, Sasako M, et al. Extended lymph-node dissection for gastric cancer. N Engl J Med 1999;340:908-14.

15. Songun I, Putter H, Kranenbarg EM, et al. Surgical treatment of gastric cancer: 15-year follow-up results of the randomised nationwide Dutch D1D2 trial. Lancet Oncol 2010;11:439-49.

16. Cuschieri A, Fayers P, Fielding J, et al. Postoperative morbidity and mortality after D1 and D2 resections for gastric cancer: preliminary results of the MRC randomised controlled surgical trial. The Surgical Cooperative Group. Lancet 1996;347:995-9.

17. Cuschieri A, Weeden S, Fielding J, et al. Patient survival after D1 and D2 resections for gastric cancer: long-term results of the MRC randomized surgical trial. Surgical Cooperative Group. Br J Cancer 1999;79:1522-30.

18. Sano T, Sasako M, Yamamoto S, et al. Gastric cancer surgery: morbidity and mortality results from a prospective randomized controlled trial comparing D2 and extended para-aortic lymphadenectomy--Japan Clinical Oncology Group study 9501. J Clin Oncol 2004;22:2767-73.

19. Sasako M, Sano T, Yamamoto S, et al. D2 lymphadenectomy alone or with para-aortic nodal dissection for gastric cancer. N Engl J Med 2008;359:453-62.

20. Degiuli M, Sasako M, Ponti A, et al. Randomized clinical trial comparing survival after D1 or D2 gastrectomy for gastric cancer. Br J Surg 2014;101:23-31.

21. Mocellin S, McCulloch P, Kazi H, et al. Extent of lymph node dissection for adenocarcinoma of the stomach. Cochrane Database Syst Rev 2015;(8):CD001964.

22. Mahar AL, McLeod RS, Kiss A, et al. A systematic review of the effect of institution and surgeon factors on surgical outcomes for gastric cancer. J Am Coll Surg 2012;214:8608.e12.

23. Hundahl SA, Phillips JL, Menck HR. The National Cancer Data Base report on poor survival of US gastric carcinoma patients treated with gastrectomy - Fifth edition American Joint Committee on Cancer staging, proximal disease, and the "different disease" hypothesis. Cancer 2000;88:921-32.

24. Bilimoria KY, Talamonti MS, Wayne JD, et al. Effect of hospital type and volume on lymph node evaluation for gastric and pancreatic cancer. Arch Surg 2008;143:671-8; 
discussion 678.

25. Gunderson LL, Sosin H. Adenocarcinoma of the stomach: areas of failure in a re-operation series (second or symptomatic look) clinicopathologic correlation and implications for adjuvant therapy. Int J Radiat Oncol Biol Phys 1982;8:1-11.

26. Macdonald JS, Smalley SR, Benedetti J, et al. Chemoradiotherapy after surgery compared with surgery alone for adenocarcinoma of the stomach or gastroesophageal junction. N Engl J Med 2001;345:725-30.

27. Smalley SR, Benedetti JK, Haller DG, et al. Updated analysis of SWOG-directed intergroup study 0116: a phase III trial of adjuvant radiochemotherapy versus observation after curative gastric cancer resection. J Clin Oncol 2012;30:2327-33.

28. Poon MA, O'Connell MJ, Moertel CG, et al. Biochemical modulation of fluorouracil: evidence of significant improvement of survival and quality of life in patients with advanced colorectal carcinoma. J Clin Oncol 1989;7:1407-18.

29. Lee J, Lim DH, Kim S, et al. Phase III trial comparing capecitabine plus cisplatin versus capecitabine plus cisplatin with concurrent capecitabine radiotherapy in completely resected gastric cancer with D2 lymph node dissection: the ARTIST trial. J Clin Oncol 2012;30:268-73.

30. Lee J, Lim DH, Kim S, et al. Phase III trial to compare capecitabine/cisplatin (XP) versus XP plus concurrent capecitabine-radiotherapy in gastric cancer (GC): The final report on the ARTIST trial. Journal of Clinical Oncology 2014;32:

31. Park SH, Zang DY, Han B, et al. ARTIST 2: Interim results of a phase III trial involving adjuvant chemotherapy and/or chemoradiotherapy after D2-gastrectomy in stage II/III gastric cancer (GC). Journal of Clinical Oncology 2019;37:

32. Cats A, Jansen EPM, van Grieken NCT, et al. Chemotherapy versus chemoradiotherapy after surgery and preoperative chemotherapy for resectable gastric cancer (CRITICS): an international, open-label, randomised phase 3 trial. Lancet Oncol 2018;19:616-28.

33. Park SH, Lee SJ, Kim ST, et al. Multicenter phase III trial of adjuvant chemoradiotherapy in stomach tumors 2 (ARTIST 2). Journal of Clinical Oncology 2015;33:

34. Park SH, Sohn TS, Lee J, et al. Phase III Trial to Compare Adjuvant Chemotherapy With Capecitabine and Cisplatin Versus Concurrent Chemoradiotherapy in Gastric Cancer: Final Report of the Adjuvant Chemoradiotherapy in Stomach Tumors Trial, Including Survival and Subset
Analyses. J Clin Oncol 2015;33:3130-6.

35. Lee J, Kang W, Lim D, et al. Phase III trial of adjuvant capecitabine/cisplatin (XP) versus capecitabine/cisplatin/ RT (XPRT) in resected gastric cancer with D2 nodal dissection (ARTIST trial): Safety analysis. Journal of Clinical Oncology 2009;27:

36. Adachi Y, Yasuda K, Inomata M, et al. Pathology and prognosis of gastric carcinoma: well versus poorly differentiated type. Cancer 2000;89:1418-24.

37. Sasako M, Sakuramoto S, Katai H, et al. Five-year outcomes of a randomized phase III trial comparing adjuvant chemotherapy with S-1 versus surgery alone in stage II or III gastric cancer. J Clin Oncol 2011;29:4387-93.

38. Cats A, Sikorska K, Verheij M. Adjuvant therapy in resectable gastric cancer-the CRITICS trial - Authors' reply. Lancet Oncol 2018;19:e331.

39. Sano T, Sasako M, Mizusawa J, et al. Randomized Controlled Trial to Evaluate Splenectomy in Total Gastrectomy for Proximal Gastric Carcinoma. Ann Surg 2017;265:277-83.

40. Cunningham D, Allum WH, Stenning SP, et al. Perioperative chemotherapy versus surgery alone for resectable gastroesophageal cancer. $\mathrm{N}$ Engl J Med 2006;355:11-20.

41. !!! INVALID CITATION !!!;

42. Patel A, Gupta VG. Adjuvant therapy in resectable gastric cancer-the CRITICS trial. Lancet Oncol 2018;19:e328.

43. Cartwright E, Keane FK, Enzinger PC, et al. Is There a Precise Adjuvant Therapy for Esophagogastric Carcinoma? Am Soc Clin Oncol Educ Book 2018;38:280-91.

44. Sauer R, Becker H, Hohenberger W, et al. Preoperative versus postoperative chemoradiotherapy for rectal cancer. N Engl J Med 2004;351:1731-40.

45. Fok M, Sham JS, Choy D, et al. Postoperative radiotherapy for carcinoma of the esophagus: a prospective, randomized controlled study. Surgery 1993;113:138-47.

46. Ténière P, Hay JM, Fingerhut A, et al. Postoperative radiation therapy does not increase survival after curative resection for squamous cell carcinoma of the middle and lower esophagus as shown by a multicenter controlled trial. French University Association for Surgical Research. Surg Gynecol Obstet 1991;173:123-30.

47. van Hagen P, Hulshof MC, van Lanschot JJ, et al. Preoperative chemoradiotherapy for esophageal or junctional cancer. N Engl J Med 2012;366:2074-84.

48. Slagter AE, Jansen EPM, van Laarhoven HWM, et al. CRITICS-II: a multicentre randomised phase II trial of neo-adjuvant chemotherapy followed by surgery 
versus neo-adjuvant chemotherapy and subsequent chemoradiotherapy followed by surgery versus neoadjuvant chemoradiotherapy followed by surgery in resectable gastric cancer. BMC Cancer 2018;18:877.

49. Stahl M, Walz MK, Riera-Knorrenschild J, et al.

Preoperative chemotherapy versus chemoradiotherapy in locally advanced adenocarcinomas of the oesophagogastric junction (POET): Long-term results of a controlled randomised trial. Eur J Cancer 2017;81:183-90.

50. Stahl M, Walz MK, Stuschke M, et al. Phase III comparison of preoperative chemotherapy compared with chemoradiotherapy in patients with locally advanced adenocarcinoma of the esophagogastric junction. J Clin Oncol 2009;27:851-6.

51. Burmeister BH, Thomas JM, Burmeister EA, et al. Is concurrent radiation therapy required in patients receiving preoperative chemotherapy for adenocarcinoma of the oesophagus? A randomised phase II trial. Eur J Cancer 2011;47:354-60.

52. Klevebro F, Alexandersson von Döbeln G, Wang N, et al. A randomized clinical trial of neoadjuvant chemotherapy versus neoadjuvant chemoradiotherapy for cancer of the oesophagus or gastro-oesophageal junction. Ann Oncol 2016;27:660-7.

53. Stiles BM, Altorki NK. The NeoRes trial: questioning the benefit of radiation therapy as part of neoadjuvant therapy for esophageal adenocarcinoma. J Thorac Dis 2017;9:3465-8.

54. Mokdad A, Ali A, Nassour I, et al. Treatment trends in gastroesophageal and gastric cancers in the United States. Journal of Clinical Oncology 2017;35:

55. Ajani JA, Winter K, Okawara GS, et al. Phase II trial of preoperative chemoradiation in patients with localized gastric adenocarcinoma (RTOG 9904): quality of combined modality therapy and pathologic response. J Clin Oncol 2006;24:3953-8.

56. Trip AK, Poppema BJ, van Berge Henegouwen MI, et al. Preoperative chemoradiotherapy in locally advanced gastric cancer, a phase I/II feasibility and efficacy study. Radiother Oncol 2014;112:284-8.

57. Ajani JA, Mansfield PF, Crane CH, et al. Paclitaxel-based chemoradiotherapy in localized gastric carcinoma: degree of pathologic response and not clinical parameters dictated patient outcome. J Clin Oncol 2005;23:1237-44.

58. Ajani JA, Mansfield PF, Janjan N, et al. Multi-institutional trial of preoperative chemoradiotherapy in patients with potentially resectable gastric carcinoma. J Clin Oncol 2004;22:2774-80.
59. Lowy AM, Feig BW, Janjan N, et al. A pilot study of preoperative chemoradiotherapy for resectable gastric cancer. Ann Surg Oncol 2001;8:519-24.

60. Allal AS, Zwahlen D, Bründler MA, et al. Neoadjuvant radiochemotherapy for locally advanced gastric cancer: long-term results of a phase I trial. Int J Radiat Oncol Biol Phys 2005;63:1286-9.

61. Wydma ski J, Suwinski R, Poltorak S, et al. The tolerance and efficacy of preoperative chemoradiotherapy followed by gastrectomy in operable gastric cancer, a phase II study. Radiother Oncol 2007;82:132-6.

62. Badgwell B, Ajani J, Blum M, et al. Postoperative Morbidity and Mortality Rates are Not Increased for Patients with Gastric and Gastroesophageal Cancer Who Undergo Preoperative Chemoradiation Therapy. Ann Surg Oncol 2016;23:156-62.

63. Ikoma N, Blum M, Estrella J, et al. Preoperative Chemoradiation Therapy Does Not Increase Risk of Anastomotic Leak in Gastric Cancer Patients. Gastroenterology 2017;152:S1217-S.

64. Badgwell B, Blum M, Elimova E, et al. Frequency of Resection After Preoperative Chemotherapy or Chemoradiotherapy for Gastric Adenocarcinoma. Ann Surg Oncol 2016;23:1948-55.

65. Badgwell B, Blum M, Estrella J, et al. Predictors of Survival in Patients with Resectable Gastric Cancer Treated with Preoperative Chemoradiation Therapy and Gastrectomy. J Am Coll Surg 2015;221:83-90.

66. Stark AP, Ikoma N, Chiang YJ, et al. Characteristics and Survival of Gastric Cancer Patients with Pathologic Complete Response to Preoperative Therapy. Ann Surg Oncol 2019;26:3602-10.

67. Ikoma N, Estrella JS, Hofstetter W, et al. Nodal Downstaging in Gastric Cancer Patients: Promising Survival if ypN0 is Achieved. Ann Surg Oncol 2018;25:2012-7.

68. Al-Batran SE, Homann N, Pauligk C, et al. Perioperative chemotherapy with fluorouracil plus leucovorin, oxaliplatin, and docetaxel versus fluorouracil or capecitabine plus cisplatin and epirubicin for locally advanced, resectable gastric or gastro-oesophageal junction adenocarcinoma (FLOT4): a randomised, phase 2/3 trial. Lancet 2019;393:1948-57.

69. Leong T, Smithers BM, Haustermans K, et al. TOPGEAR: A Randomized, Phase III Trial of Perioperative ECF Chemotherapy with or Without Preoperative Chemoradiation for Resectable Gastric Cancer: Interim Results from an International, Intergroup 
Trial of the AGITG, TROG, EORTC and CCTG. Ann Surg Oncol 2017;24:2252-8.

70. Leong T, Smithers BM, Michael M, et al. TOPGEAR: a randomised phase III trial of perioperative ECF chemotherapy versus preoperative chemoradiation plus perioperative ECF chemotherapy for resectable gastric cancer (an international, intergroup trial of the AGITG/TROG/EORTC/NCIC CTG). BMC Cancer 2015;15:532.

71. Hoeppner J, Lordick F, Brunner T, et al. ESOPEC: prospective randomized controlled multicenter phase III trial comparing perioperative chemotherapy (FLOT protocol) to neoadjuvant chemoradiation (CROSS protocol) in patients with adenocarcinoma of the esophagus (NCT02509286). BMC Cancer 2016;16:503.

72. Moningi S, Ajani JA, Badgwell BD, et al. IMRT Reduces Acute Toxicity in Patients Treated With Preoperative Chemoradiation for Gastric Cancer. Adv Radiat Oncol 2020;5:369-76.

73. Schernberg A, Rivin Del Campo E, Rousseau B, et al. Adjuvant chemoradiation for gastric carcinoma: State of the art and perspectives. Clin Transl Radiat Oncol 2018;10:13-22.

74. Zhang N, Fei Q, Gu J, et al. Progress of preoperative and postoperative radiotherapy in gastric cancer. World J Surg Oncol 2018;16:187.

75. Shinde A, Novak J, Amini A, et al. The evolving role of radiation therapy for resectable and unresectable gastric cancer. Transl Gastroenterol Hepatol 2019;4:64.

76. Chakravarty T, Crane CH, Ajani JA, et al. Intensitymodulated radiation therapy with concurrent chemotherapy as preoperative treatment for localized gastric adenocarcinoma. Int J Radiat Oncol Biol Phys

doi: $10.21037 / \operatorname{tgh} .2020 .03 .01$

Cite this article as: Ludmir EB, Das P. Shifting sands: the role of radiotherapy for patients with gastric and gastroesophageal adenocarcinoma. Transl Gastroenterol Hepatol 2021;6:50.
2012;83:581-6.

77. Dahele M, Skinner M, Schultz B, et al. Adjuvant radiotherapy for gastric cancer: A dosimetric comparison of 3-dimensional conformal radiotherapy, tomotherapy and conventional intensity modulated radiotherapy treatment plans. Med Dosim 2010;35:115-21.

78. Ringash J, Perkins G, Brierley J, et al. IMRT for adjuvant radiation in gastric cancer: a preferred plan? Int J Radiat Oncol Biol Phys 2005;63:732-8.

79. Alani S, Soyfer V, Strauss N, et al. Limited advantages of intensity-modulated radiotherapy over $3 \mathrm{D}$ conformal radiation therapy in the adjuvant management of gastric cancer. Int J Radiat Oncol Biol Phys 2009;74:562-6.

80. Minn AY, Hsu A, La T, et al. Comparison of intensitymodulated radiotherapy and 3-dimensional conformal radiotherapy as adjuvant therapy for gastric cancer. Cancer 2010;116:3943-52.

81. Trip AK, Nijkamp J, van Tinteren H, et al. IMRT limits nephrotoxicity after chemoradiotherapy for gastric cancer. Radiother Oncol 2014;112:289-94.

82. Oppedijk V, van der Gaast A, van Lanschot JJ, et al. Patterns of recurrence after surgery alone versus preoperative chemoradiotherapy and surgery in the CROSS trials. J Clin Oncol 2014;32:385-91.

83. Conrad T, MacLellan S, Kassam Z, et al. Retrospective assessment of patterns of recurrence relative to radiotherapy volumes for adjuvant conformal chemoradiotherapy in gastric cancer. Gastric Cancer 2016;19:887-93.

84. Elimova E, Slack RS, Chen HC, et al. Patterns of relapse in patients with localized gastric adenocarcinoma who had surgery with or without adjunctive therapy: costs and effectiveness of surveillance. Oncotarget 2017;8:81430-40. 